\title{
HARMONIZAÇÃO DO SORRISO POR MEIO DE CIRURGIA PERIODONTAL E RESTAURAÇÕES DIRETAS EM RESINA COMPOSTA: RELATO DE CASO
}

\author{
SMILE HARMONIZATION BY MEANS OF PERIODONTAL SURGERY AND \\ DIRECT RESTORATION IN COMPOSITE RESIN: CASE REPORT
}

\author{
Gustavo Martins GONTIJO'; João GONTIJO NETO'; Kleber Vinícius Rodrigues dos SANTOS²; João Batista de SOUZA3; \\ Arioldo Teles de CASTRO ${ }^{4}$ \\ 1 - Cirurgião-dentista pela Faculdade de Odontologia da Universidade Federal de Goiás. \\ 2 - Mestrando em Clínica Odontológica pela Faculdade de Odontologia da Universidade Federal de Goiás. \\ 3 - Professor titular da disciplina de Dentística da Universidade Federal de Goiás. \\ 4 - Professor titular da disciplina de Periodontia da Universidade Federal de Goiás.
}

\section{RESUMO}

Procedimentos estéticos odontológicos estão cada vez mais integrados com outras áreas, trabalhando em prol da otimização de resultados psicossociais e funcionais, e aumentando as possibilidades inclusas no plano de tratamento. A estética apresenta grande relevância na odontologia moderna, a demanda por sorrisos brancos e harmoniosos aumenta proporcionalmente às novas técnicas existentes. O presente trabalho objetiva relatar um caso de reabilitação estética a partir do planejamento digital do sorriso que dará informações benéficas para avaliar as diversas possibilidades frente ao segmento restaurador e periodontal cirúrgico. Paciente R. C. C., 22 anos, sexo masculino, compareceu a clínica integrada com queixa estética nos dentes anteriores. A partir do exame clínico inicial, foram solicitados exames de rotina concomitantemente à moldagem para modelo de estudo. Após o estudo de caso, esclarecimento do planejamento ao paciente e frente as expectativas estéticas, foi solicitado o exame de Planejamento Digital do Sorriso (Digital Smile Design - DSD). Foi realizada a cirurgia de gengivoplastia e osteoplastia dos pré-molares nos dentes 12, 14, 15, 24 e 25, fundamentada nos parâmetros fornecidos pelo exame digital e restaurações diretas em resina composta após o período de cicatrização adequado. Cada vez mais, é exigido do cirurgião-dentista, uma visão ampla que permita explorar as diversas perspectivas vigentes atendendo aos interesses e necessidades do paciente. Conclui-se, independentemente da área da atuação, a realização e otimização de resultados caminha lado a lado com a multidisciplinariedade que, a cada dia que se passa, ganha importância na formação acadêmica profissional.

PALAVRAS-CHAVE: Resinas compostas; Estética Dentária; Gengivoplastia.

\section{INTRODUÇÃO}

A preocupação com a estética vem se propagando e tomando proporções cada vez mais evidentes no mundo atual ${ }^{1}$. A odontologia, por sua vez, proporciona sorrisos cada vez mais brancos e simétricos em busca da adequação perfeita entre fatores que extrapolam aquilo que é meramente visto a olho nu.

A tecnologia, visando aperfeiçoar os planos de tratamento e suas técnicas transcirúrgicas, tem se manifestado de maneira cada vez mais engrandecedora. A tecnologia de Planejamento Digital do Sorriso (Digital Smile Design - DSD) tem sido utilizada para facilitar os procedimentos estéticos e permitir uma melhor visão diagnóstica, melhor comunicação entre os membros da equipe e melhor previsão dos resultados esperados ${ }^{2}$.

Alguns estudos mencionaram sobre a relação entre o tecido periodontal e restaurações marginais revelando também que dentes com coroas clínicas curtas possuíam indicação para a cirurgia de aumento de coroa. Portanto, os dentes localizados na região anterossuperior receberam maior consideração frente à presença de sorriso gengival ${ }^{3}$.
Graças ao advento da tecnologia adesiva e à consistente melhoria das propriedades dos materiais, dentes anteriores comprometidos podem ser satisfatoriamente restaurados com resina composta ${ }^{4}$. E uma das melhores vantagens no uso de resina composta, está relacionada com o ótimo resultado estético que podem proporcionar, pois é possível recriar uma aparência natural próxima às estruturas dentais, restaurando a função morfológica e restabelecendo características como cor, translucidez, matiz, croma e valor ${ }^{5}$.

Assim, é evidenciada, a importância que a condição de saúde bucal possui na qualidade de vida dos indivíduos, e imprescindível um estudo integrado, não apenas para proporcionar estética, mas também para levar em consideração os resultados funcionais, psicossociais, melhores condições de saúde bucal e qualidade de vida ${ }^{6,7,8}$. Desta forma, este trabalho procura relatar um caso clínico onde houve a associação da periodontia a dentística no sucesso da harmonização do sorriso. 


\section{MATERIAL E MÉTODO}

Paciente, RCC, 22 anos, sexo masculino, compareceu à clínica integrada da Faculdade de Odontologia da Universidade Federal de Goiás (FO/UFG) com queixa principal de "meus dentes são muito curtos", após o exame clínico, foi entregue um pedido de exames complementares onde é possível diagnosticar e tratar com melhor precisão a queixa estética (Figura 1).

A partir do exame clínico inicial, foi realizado uma fotografia do sorriso (Figura 2) e foram solicitados exames de rotina concomitantemente à moldagem para confecção do modelo de estudo. Após o estudo de caso e esclarecimento do planejamento ao paciente, frente as expectativas estéticas, foi solicitado o exame de análise de sorriso DSD.

Com a análise do sorriso DSD juntamente com a impressão 3D da arcada superior para estudo (Figura 3), foi possível a realização do planejamento cirúrgico periodontal e estético dental, chegando ao plano de tratamento que inclui a gengivoplastia nos dentes 12, 14, 15, 24 e 25 (Figura 4) e fechamendo de diastemas anterossuperiores.

Para a cirurgia de aumento de coroa foram selecionados os dentes $(12,14,15,16,24$, 25 e 26), desta forma, foram realizadas anestesias dos nervos alveolares superiores posterior, médio e anterior, juntamente com anestesia do nervo palatino maior e infiltrativas. A técnica utilizada para estabelecer o aumento e contorno coronário foi a técnica de incisão em bisel interno, manuseando o cabo de bisturi e lâmina 15C (Figura 5).

Logo em seguida, foi realizado nos dentes 13 ao 16 e 23 ao 26, o afastamento dos retalhos com deslocador de periósteo Molt e a osteotomia do osso alveolar, por vestibular, manuseando caneta de alta rotação e broca diamantada 1014 HL, Em seguida, foi executado a sutura interpapilar utilizando porta agulha com vídea e fio de sutura 4-0 de seda (Figura 6).

Sete dias após a cirurgia de aumento de coroa, foram retiradas as suturas obtendo um ótimo aumento e contorno gengival (Figura 7). Para um melhor prognóstico, o paciente teve um intervalo mínimo de 60 dias entre o aumento de coroa e a realização das restaurações diretas em resina composta.

Após o período de maturação tecidual, foi realizado então, as restaurações diretas em resina composta com o intuíto de fechar os diastemas que o paciente possuia entres os dentes (12 e 13) e (22 e 23), melhorando a estética e harmonia do sorriso. Desta forma, realizou-se a profilaxia destes elementos dentários, foi selecionada a cor da resina composta (A1) confeccionado o bisel com broca diamantada n. 1111, e foi inserido o fio retrator 00 para afastamento gengival e controle de umidade do campo operatório proporcionando a confecção da restauração (Figura 8).

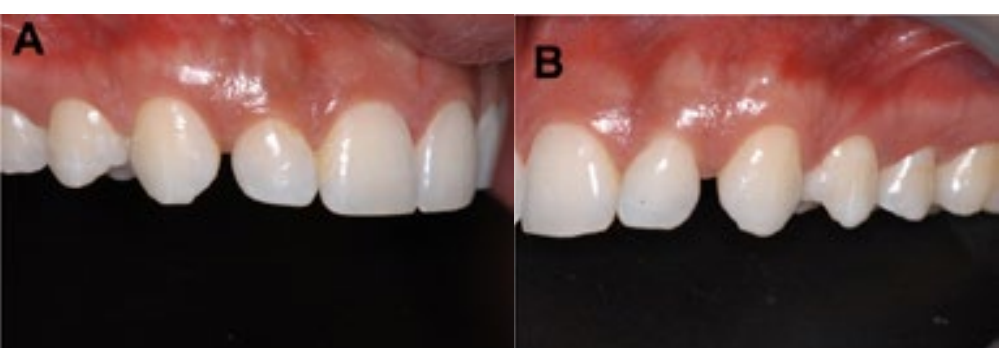

Figura 4A - Visão vestibular direita de região anterossuperior; B - Visão vestibular esquerda da região anterossuperior.

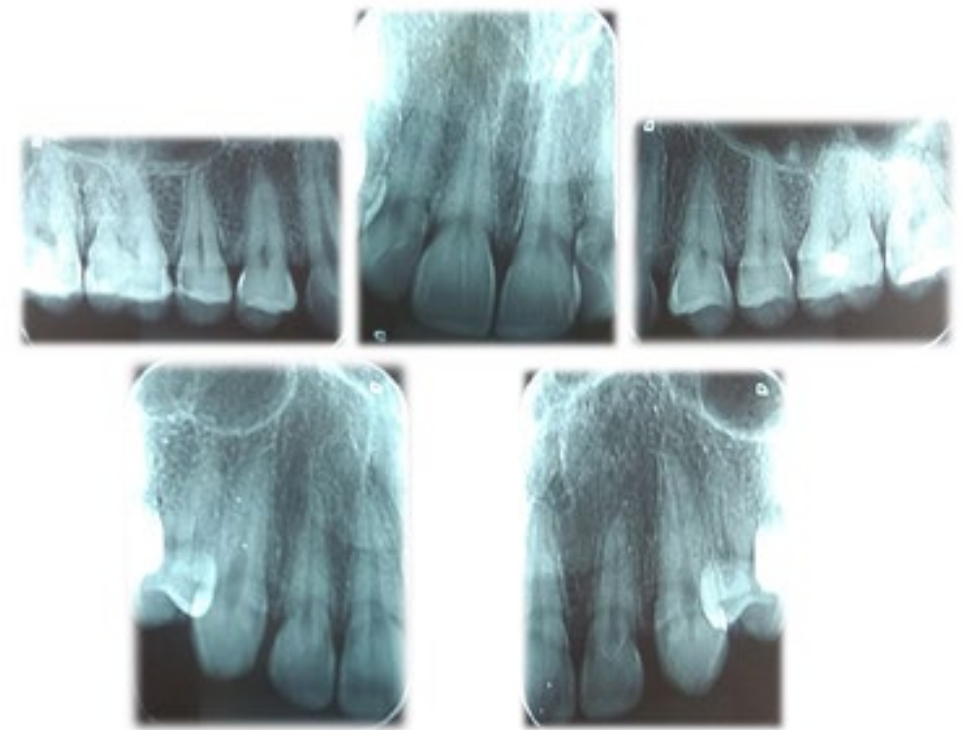

Figura 1 - Radiografias periapicais solicitadas após exame clínico.

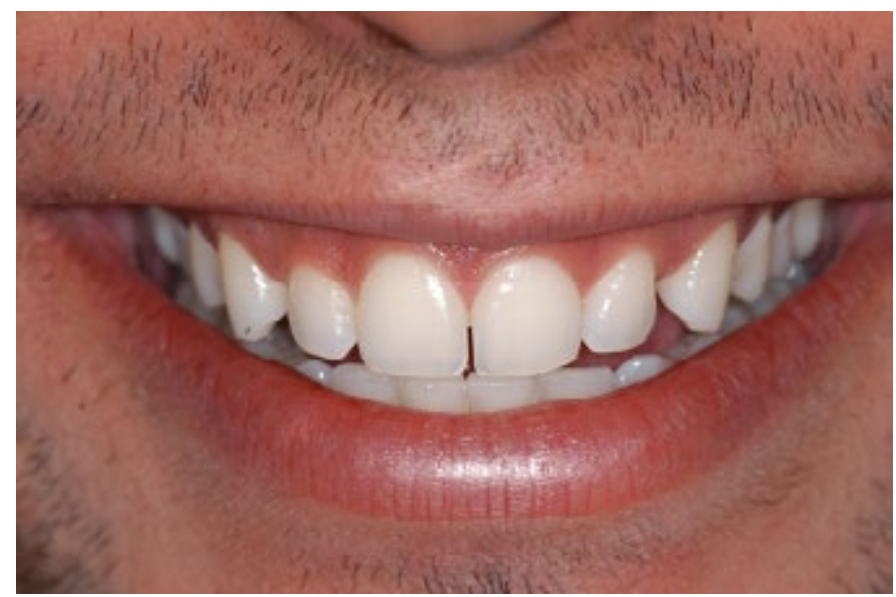

Figura 2 - Fotografia inicial da linha de sorriso.

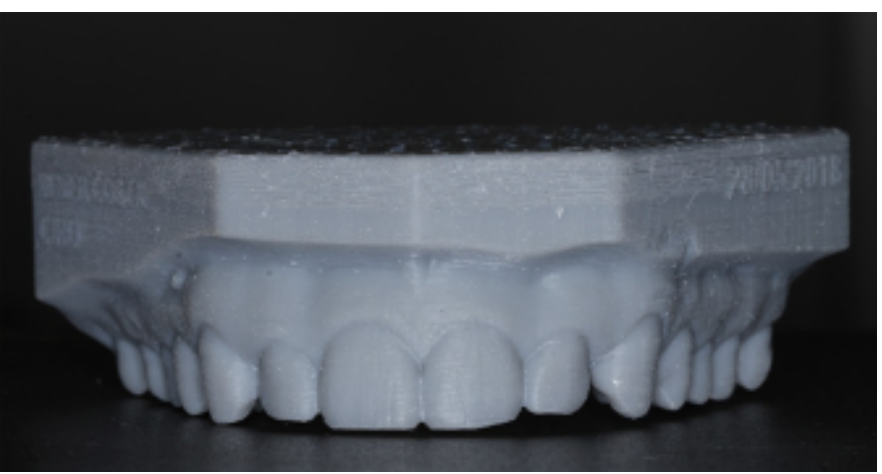

Figura 3 - Modelo de estudo 3D.

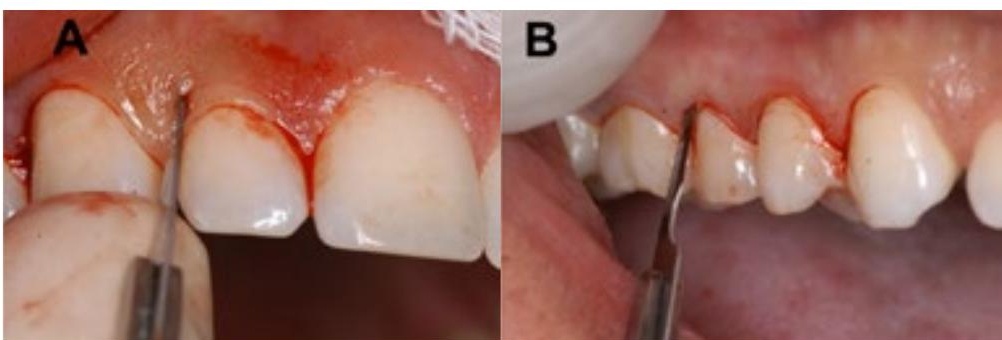

Figura 5A - Técnica de incisão em bisel interno; B - Manuseio da lâmina de bisturi 15C nos dentes 14 e 15. 
Posteriormente, os dentes 12, 13, 22 e 23 foram condicionados com ácido fosfórico a $37 \%$ por 30 segundos, lavando-os com água pelo dobro do tempo. Logo após a secagem com bolinha de algodão, foi aplicado o sistema adesivo (duas camadas, com leve jato de ar entre elas por 20 segundos) com microaplicadores. Em seguida foi realizada fotopolimerização do sistema adesivo por 20 segundos (Figura 9).

Com os dentes condicionados e preparados para confeccionar as restaurações diretas em resina composta, realizou-se incrementos de resina com espátula para inserção, seguidos de fotopolimerizações de 20 segundos entre cada incremento (Figura 10). Em seguida, foi complementado o posicionamento final da resina com pincel pelo de marta, seguido de fotopolimerização por 40 segundos e verificação do contato oclusal com tira de papel carbono Accufilm (Figura 11).

Sete dias após a confecção das restaurações, o paciente retornou a clínica para efetuar o polimento dos dentes restaurados, com escova de carbeto de silício e disco de feltro com a pasta de polimento para obter o brilho final (Figura 11).

Assim, a harmonização das restaurações diretas em resinas compostas, juntamente com a cirurgia periodontal de aumento de coroa, trouxeram mais equilíbrio e, consequentemente, um ótimo resultado estético (Figura 12).

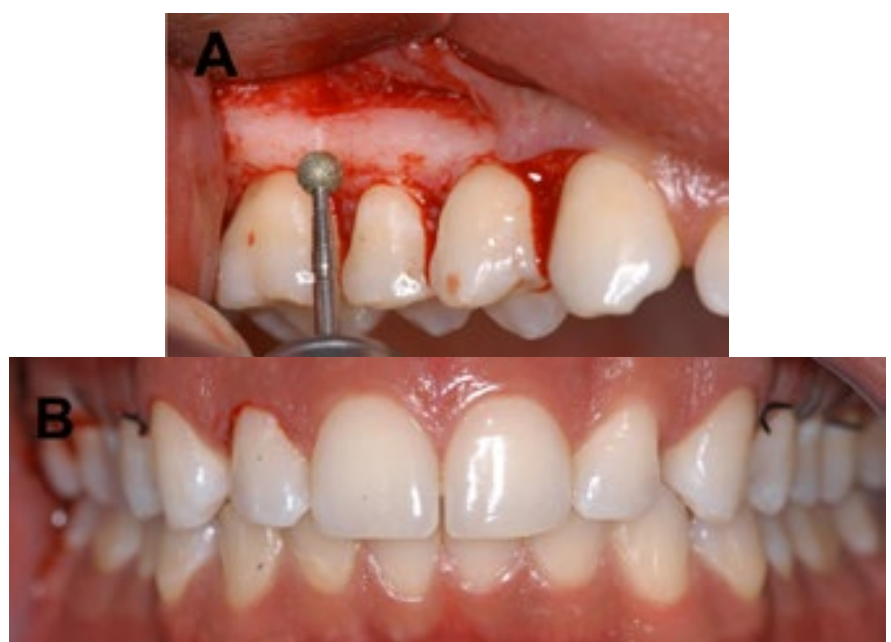

Figura 6A - Osteotomia alveolar vestibular após realização de retalho; B - Sutura interpapilar com fio de seda 4-0, pós operatório imediato.

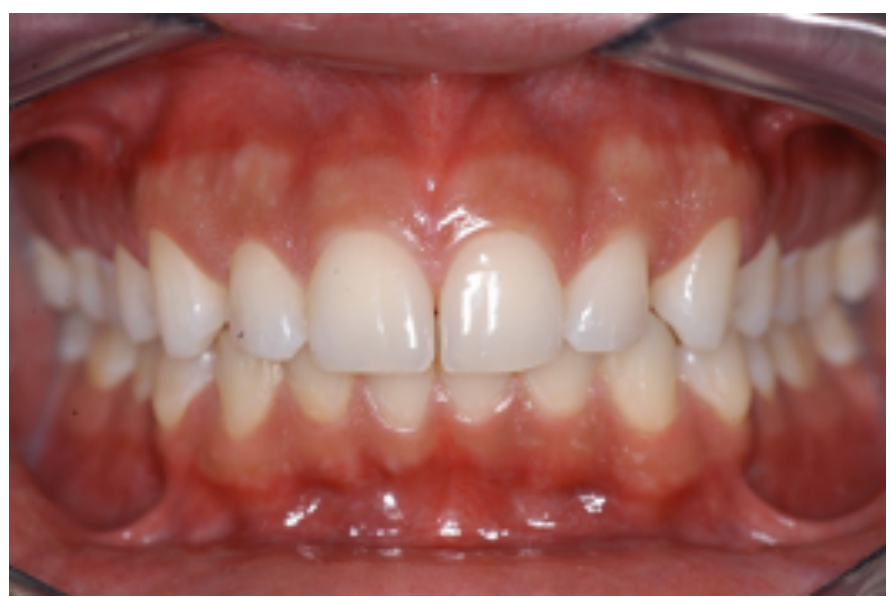

Figura 7 - Retirada de suturas e 1 semana de pós operatório.

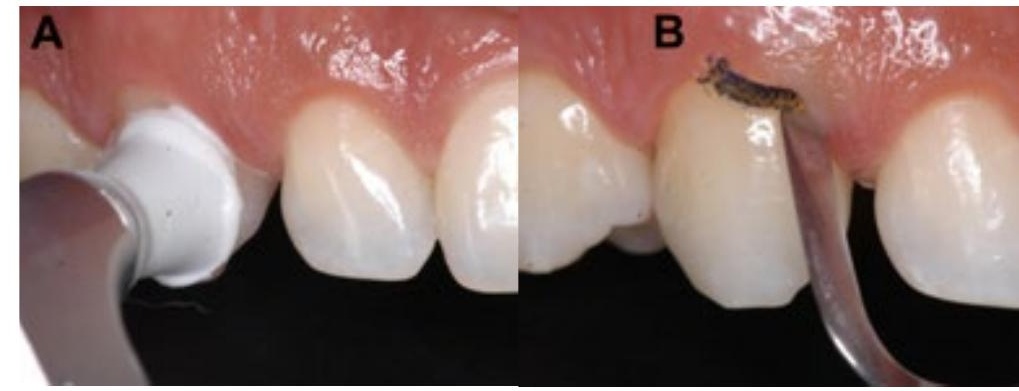

Figura 8A - Profilaxia; B - Inserção do fio retrator 2-0

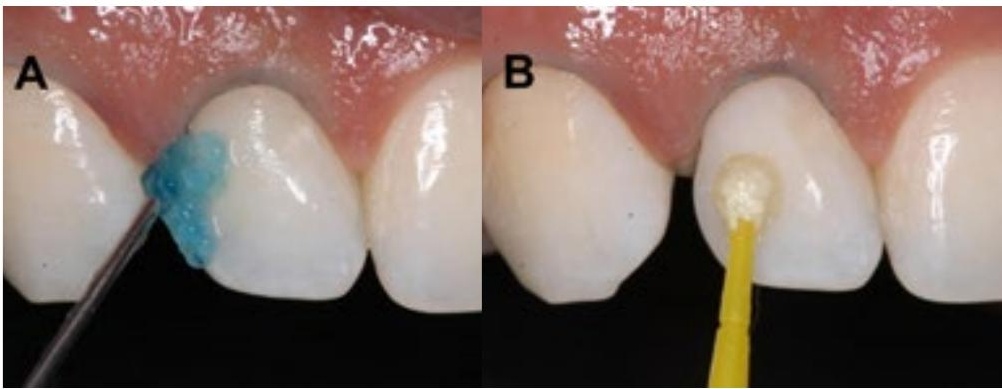

Figura 9A- Aplicação do ácido fosfórico 37\%; B - Aplicação do sistema adesivo

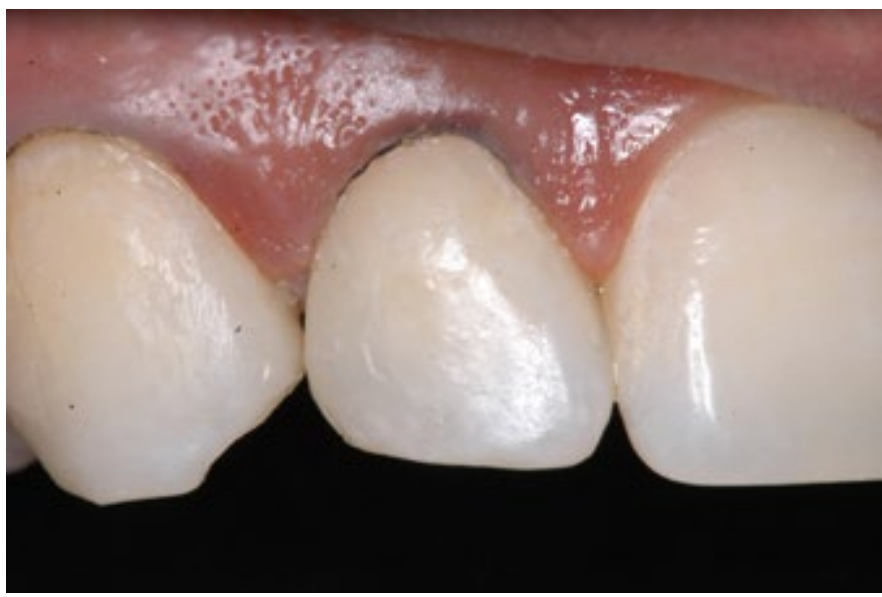

Figura 10 - Aspecto imediato final após a restauração em resina direta.

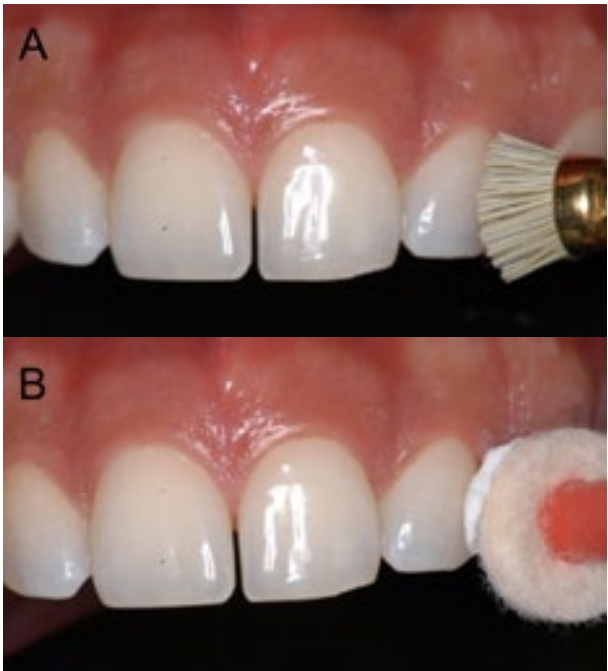

Figura 11A - Polimento com escova de carbeto de silício; B - Polimento com disco de feltro e pasta de polimento Diamond. 


\section{DISCUSSÃO}

Cada vez mais, os pacientes apresentam queixas estéticas onde é necessário uma avaliação profunda sobre os campos macro e micro estéticos, exigindo mais ainda do profissional que apresenta a necessidade de constante aprendizado frente às diversas possibilidades clínico-cirúrgicas ${ }^{1,2,9,10}$.

Nesse relato de caso, o paciente apresentou queixa estética onde foi possível o planejamento à partir de diversas vertantes e/ou filosofias de reabilitação. O uso de laminados cerâmicos, a realização de aumento incisal, a intervenção cirúrgica periodontal envolvendo os dentes selecionados; Foram alvos de discussão e a tomada de decisões foi acrescida de fatores exclusivos do paciente ${ }^{1,2,11}$.

Quanto às possibilidades de tratamento, a utilização de acréscimo por resina composta mostrou-se como a melhor opção, levando em consideração a exclusiva necessidade de fechamento dos diastemas entre incisivos laterais e caninos e a ausência da necessidade de aumento incisal, sendo, a altura vertical do dente, controlada pelo procedimento periodontal ${ }^{11-15}$.

Levando em consideração o tamanho das raízes anteriores, o procedimento de gengivoplastia definiu-se como limitado mediante à abordagem conservadora, sendo mais ampla na região posterior do sorriso. Concomitante à osteotomia posterior, houve uma melhor adequação da gengiva no novo tecido ósseo de suporte $^{3,11,12}$.

O acompanhamento clínico é essencial para avaliar o sucesso do tratamento, e equilíbrio das relações mésio-distal, nesse caso, o paciente está ciente das condições em que foi posto. Novas avaliações necessitam ser realizadas, para o bom prognóstico ${ }^{4,13}$.

\section{CONCLUSÃO}

A abordagem multidisciplinar envolvendo as áreas de dentística e periodontia cirúrgica, como no presente relato de caso, apresentou-se como uma boa opção para o planejamento e execução de uma queixa apenas estética do paciente, otimizando resultados e agregando valor à prática odontológica. Assim, proporcionou-se a harmonia dentofacial e a satisfação do paciente com o resultado obtido.

\section{REFERÊNCIAS}

01. Suzuki L, Machado AW, Bittencourt MAV. Avaliação da influência da qualidade de exposição gengival na estética do sorriso. Dental Press J Orthod. 2011; 16(5): 1-10.

02. Coachman C, Ricci A, Calamita M, Yoshinaga LG. Desenho digital do sorriso: do plano de tratamento à realidade clínica. In: Paolucci B, et al. Visagismo: a arte de personalizar o desenho do sorriso. São Paulo: Vm Cultural; 2011. p. 147-162.

03. Padbury A Jr, Eber R, Wang HL. Interactions between the gingiva and the margin of restorations. J Clin Periodontol. 2003; 30(5): 379-85.

04. Gondo R, Marson FC, Alvares I. Restaurações diretas de resina composta em dentes anteriores: alguns fundamentos para a obtenção de resultados estéticos satisfatórios. Clín Int J Braz Dent. 2005;1(2):137-145.

05. Schwarz V, Simon LS, Silva SA, Ghiggi PC, Cericato GO. Fechamento de diastema com resina composta: relato de caso. J oral invest. 2013; 2(1): 26-31.

06. Davis DM, Fiske J, Scott B, Radford DR. The emotional effects of tooth loss: a preliminary quantitative study. Br Dent J. 2000;188(9):503-6.

07. Fiske J, Davis DM, Frances C, Gelbier S. The emotional effects of tooth loss in edentulous people. Br Dent J. 1998;184(2):90-3.

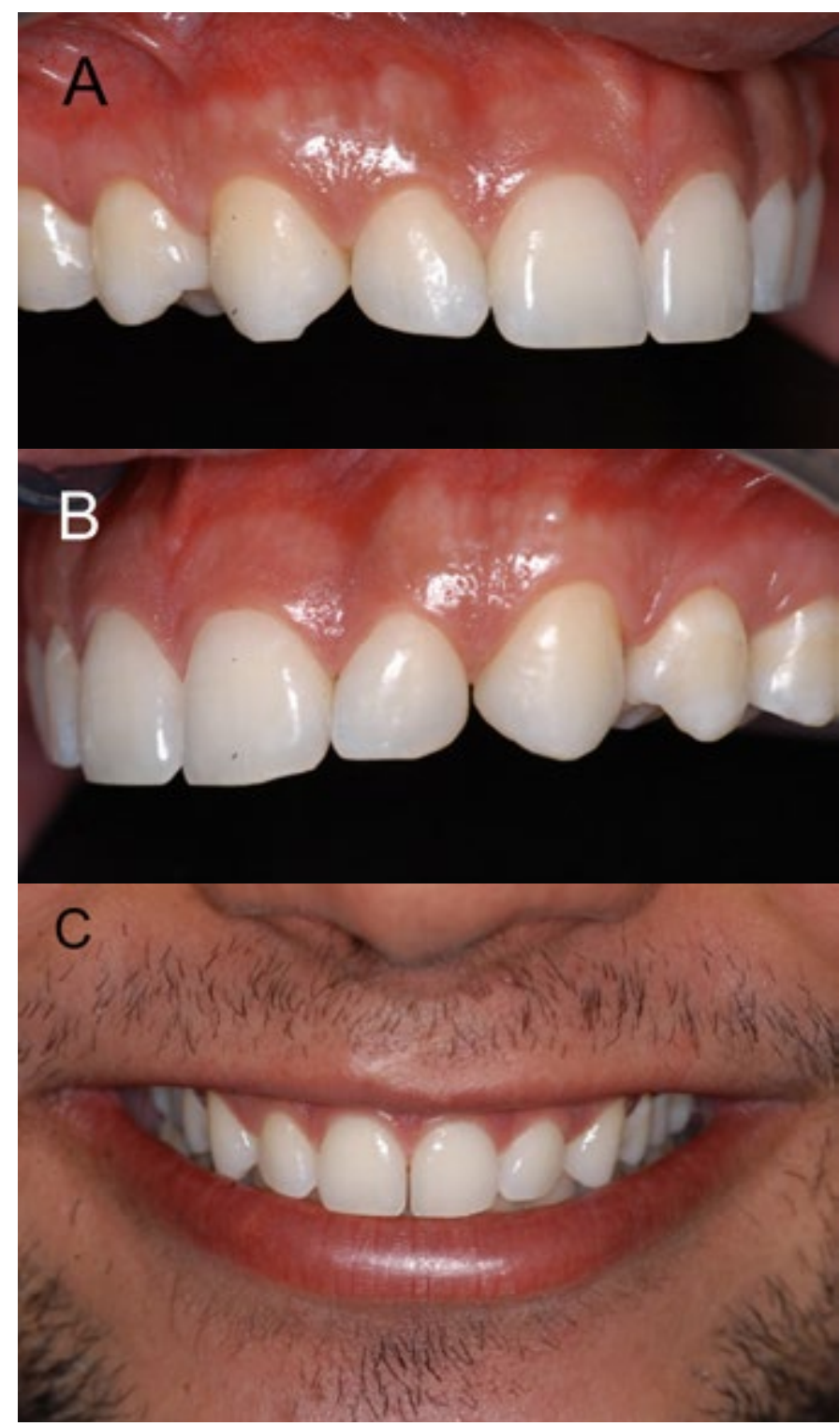

Figura 12A - Vista anterossuperior direita; B - Vista anterossuperior esquerda C - Sorriso final do paciente.

08. Krall E, Hayes C, Garcia R. How dentition status and masticatory function affect nutrient intake. J Am Dent Assoc. 1998; 129(9):1261-9.

09. Coachman C, Calamita M, Schyder A. Digital smile design: uma ferramenta para planejamento e comunicação em odontologia estética. Dicas. 2012; 1(2): 36-41.

10. Khuller N, Sharma N. Biological Width: Evaluation and Correction of its Violation. Journal of Oral Health \& Community Dentistry. 2009; 3(1): 20-25.

11. Ong M, Tseng S, Wang H. Crown Lengthening Revisited. Clinical Advances in Periodontics. 2011; 1(3): 233-239.

12.12 Mangani F, Cerutti A, Putignano A, Bollero R, Madini L. Clinical approach to anterior adhesive restorations using resin composite veneers. Eur J Esthet Dent. 2007; 2(2): 188-209.

13. Silva AF, Lund RG. Dentística restauradora: do planejamento à execução. 1. ed. Rio de Janeiro: Editora Santos; 2016.

14. Soares LM, Soares C. Resultados previsíveis no uso de laminados e fragmentos cerâmicos com preparo minimamente invasivos. Clinica Int J Braz Dent. 2011; 7(1):36-50. 


\section{ABSTRACT}

Aesthetic dental procedures are increasingly integrated with other areas, working towards the optimization of psychosocial and functional outcomes, and increasing the possibilities included in the treatment plan. Aesthetics has great relevance in modern dentistry, the demand for harmonious white smiles increases in proportion to new techniques. The present abstract aims to report a case of aesthetic rehabilitation from the digital smile planning that will give beneficial information to evaluate the various possibilities before the restorative segment and surgical periodontal. Patient R. C. C., 22 years old, male, attended the integrated clinic with aesthetic complaint in the anterior teeth. From the initial clinical examination, routine exams were requested concomitantly with the modeling of the study model. After the case study, clarification of patient planning and aesthetic expectations, the Digital Smile Design (DSD) exam was requested. Gingival surgery was performed on teeth allied to osteoplasty of the premolars 12, 14, 15, 24 and 25, based on the parameters provided by the digital examination and direct restorations in composite resin after the appropriate healing period. Increasingly, the dental surgeon is required to have a broad vision that allows exploring the diverse perspectives that are in place, taking into account the interests and needs of the patient. We can conclude that, regardless of the area of performance, the realization and optimization of results goes hand in hand with the multidisciplinarity that, every day that passes, gains importance in the professional academic formation

KEYWORDS: Composite Resins; Esthetics, Dental; Gingivoplasty.

\section{AUTOR PARA CORRESPONDÊNCIA}

Kleber Vinícius Rodrigues dos Santos

Rua N, Qd. 49, Lt. 03, Vila Santa Helena

Cep.: 74555-120, Goiânia - GO

Telefone: (62) 9 8564-0032

E-mail: klebervinicius@live.com 